\title{
Improved Energy Efficiency Through Integration of Self- Powered Sensors with Mobile Communication
}

\author{
Bernd Kärcher ${ }^{1}$, Gerd Scholl ${ }^{2}$, Dieter Mintenbeck ${ }^{3}$ \\ ${ }_{1}^{1}$ Festo $A G$ \& Co KG, Esslingen; Germany, kch@de.festo.com \\ ${ }^{2}$ Helmut Schmidt University -University of the Federal Armed Forces, Hamburg, Germany \\ ${ }^{3}$ HSG-IMIT (Institut für Mikro- und Informationstechnik), Villingen-Schwenningen, Germany
}

\begin{abstract}
:
Energy efficiency of production plants is continuously getting more public attention while at the same time, energy is the essential resource for the manufacturing industry to maintain its capacity to act.. As a consequence, measurement and monitoring of substantial parameters of the manufacturing process are essential to achieve the goal of an optimized energy consumption. On shop floor level, sensors have to be integrated to provide a representative picture of the manufacturing process.. Compared to conventional approaches, wireless and self-powered sensors can be implemented at much lower installation costs. In this paper, a new generation of integrated sensors are introduced, including technologies for energy supply and wireless communication as well as appropriate packaging and integrating technologies. Although first prototypes are realized in the field of pneumatics, electrical and/or further parameters can be measured with the modular concept proposed. Main contributions are derived from the public funded research project "MIKOA" [1]
\end{abstract}

Key words: Energy Efficeny, Self-Powered Sensors, Energy Harvesting, Pneumatic Energy Consumption, Wireless Communication.

\section{Introduction}

Facing rising energy costs and the limited availability of raw materials, the efficient use of energy is gaining more and more importance. The share of the German industry in the total consumption of electricity added up to $43 \%$ in 2010, representing a main driver for the deployment of the "High-Tech Strategy for Germany", an initiative of the Federal Government that addresses global challenges. Broken down on the industrial environment, it is necessary to analyze the demand of energy for certain production units. Therefore, selfpowered sensors are installed in crucial positions of the energy supply network. A wireless communication network helps to easily integrate the information into an existing IT infrastructure. The main target is to give as precise recommendations as possible to optimize the local energy consumption on plant level.

The concepts for the energy management of factory and process automation equipment mainly depend on the amount and quality of the available data. These data are usually provided by wired sensors, which are only available at the local end of the cable connection. This traditional design implies major restrictions in terms of flexibility and the manifold of possible application areas for components, systems and units (mobile units and moving/rotating installation components).

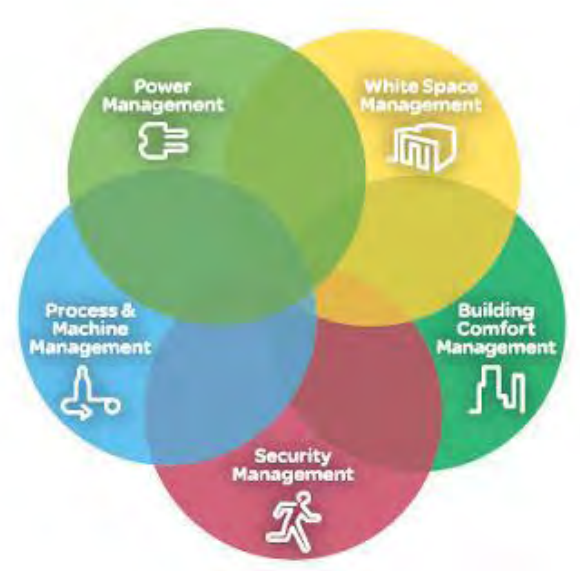

Fig. 1. Integrated information management [2].

These restrictions can be resolved by using wireless communicating components, systems and units, thus creating new possibilities for extensive system or installation diagnosis thanks to globally available and networkable information. 
As a consequence, a deeper under-standing of the overall manufacturing process acreates a potential for the optimization of costs and quality and thus the energy efficiency of a manufacturing unit is of particular importance.

In the context of competitiveness, the following factors are crucial for a successful establishment of this innovative concept:

- Realization of an interference-immune and real-time-capable wireless communication

- Development of miniaturized and maintenance-free sensor/actuator units which allow efficient operation with autonomous or mono-energy power supplies.

The development and optimization of these basic technologies and components represented the main subjects of the work of the consortium involved in the MIKOA project.

\section{Main aspects of work}

Concepts for miniaturized and wireless network components for use in factory and process automation and diagnosis are designed, implemented and verified by means of appropriate prototypes. These components are energy-autonomous sensors or sensor/actuator modules which are applied to control processes or carry out system diagnosis. Energyautonomous in this context means having a decentralised electrical power supply from ambient sources, for example kinetic process energy (impact, shock, vibration) or energy obtained from light by photovoltaic means. The potential electrical power output of these sources will generally not adequately satisfy the requirements of the described sensor or sensor/actuator modules. As a result, use is also made of unused pressure and/or flow energy of any fluidic process media present. Energy-autonomous therefore means the elimination of all wired electrical interfaces outside the system boundaries. In order to make this goal possible and at the same time to achieve a balanced energy budget, it may be appropriate to combine different types of converters and energy storage devices. This requires the development of an energy management system which firstly provides conversion of the different voltages and secondly appropriate energy distribution in accordance with overarching priorities.

In order to allow wireless communication to be realised and to achieve the necessary market acceptance for wireless solutions in competition with wired systems, the wireless solutions must be comparable to wired systems in terms of reliability, size and its ability to provide maintenance-free operation. Moreover, it has to offer significant advantages regarding installation and suitability for mobile applications.

Further development of wireless technologies is also necessary to meet the requirements of the automation sector, particularly with regard to defined real-time behavior and energy efficiency.

\section{Autonomous systems}

As shown schematically in Fig. 2, systems can be called autonomous if they have no wired energy or communications interface. Therefore it is possible, within the limits of a given component or system, to deviate from the usual standard specifications for factory or process automation, since units of this kind are intrinsically capable of being encapsulated or being produced as enclosed systems. In concrete terms, this means, for example, a free choice of internal system voltages in order to optimise the efficiency of the unit as much as possible and to reduce losses caused by voltage conversion. This also favours use in environments with higher-level requirements, for example areas with extreme temperatures or with potentially explosive atmospheres.

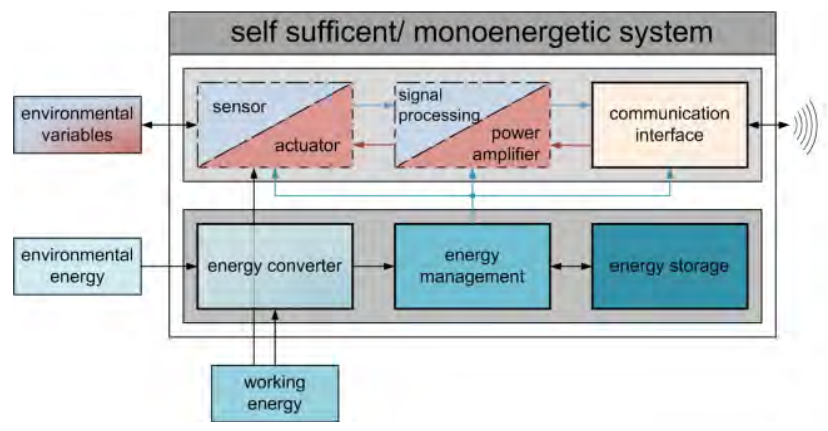

Fig. 2 Schematic representation of an autonomous system (source: Festo)

One major technical problem is, however, to ensure a reliable power supply for sensors and sensor/actuator units when boundary conditions strictly define acceptable power consumption or functionality of the unit in question. A goal conflict arises between the amount of energy that can be accumulated within a certain time window and the power consumption of the unit.

In order to develop meaningful solutions to this problem, it is necessary to conduct analysis and solution development on a holistic basis. This means, for example, that the allocation of the necessary energy/power has to be optimized at first. This can be achieved by improving the efficiency of the converters or by using several different energy sources (converter arrays). For 
example, buffering and the use of several energy sources allow to bridge periods in which particular energy sources are not available.

It is also necessary to optimize the efficiency of sensors, actuators, function modules for wireless communication and data processing components. Finally, energy management offers ways of using efficient strategies to activate or deactivate targeted components or functions.

\section{Examples of applications}

Two application cases have been defined for the realization of autonomous units. These differ significantly in terms of their requirements for wireless communication and energy supply.

In the area of plant monitoring, it is of interest to be able to determine process variables such as system pressure or air consumption by the use of a pq sensor unit, as shown in Fig. 3. These variables can be measured and transmitted in extended intervals or when explicitely triggered by state changes and are not relevant to the control system. The longer measuring-cycle intervals result in lower energy consumption. With regards to energy sources, the main focus here is on the pressure and flow energy of the medium of compressed air. This approach is highly suitable in view of the fact that pneumatic interfaces are designed for that purpose and the energy density of the compressed air offers sufficient potential for the decentral provision of electrical energy. However, to cover the case in which an adequate level of pneumatic energy is not available, for example due to system malfunctions, an additional solar cell is used to provide sufficient energy to permit low-power operation with limited communication capacity.

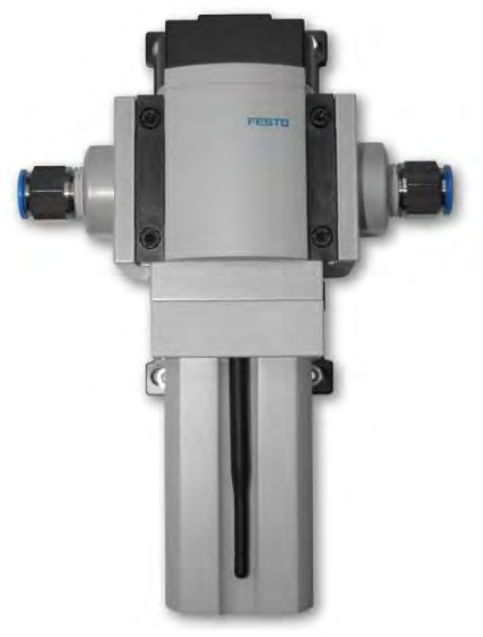

Fig. $3 \quad p-Q$ sensor unit for plant monitoring (source: Festo)
Applications involving high-speed handling systems, such as those commonly used in factory automation, are more complex in terms of energy management. The characteristic data that are determined, such as positions, pressures and flow rates, are highly relevant for the control system and are measured and transmitted with a high sampling rate. This requires reliable transmission and wireless communication with deterministic and real-time capabilities.

These stringent time and reliability requirements turn into a relatively high power consumption which normally cannot be met by familiar energy-harvesting concepts such as thermal converters, vibration converters or solar cells. For this reason, once again the emphasis is on the exploitation of energy from the medium in combination with a solar cell.

For this application case, too, a pq sensor unit has been developed which retrieves the residual energy in the exhaust air of individual pneumatic drives. The emphasis here is not only on the energy requirements already described but also on the miniaturization of the overall sensor unit, as shown in Fig. 4.

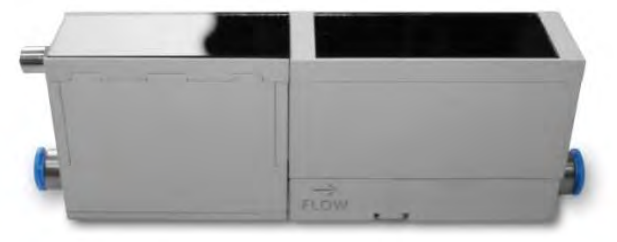

Fig. 4 Miniaturised $p-Q$ sensor units (source: Festo)

This kind of diagnostic and monitoring functionality can also be used for handling systems. It may therefore be appropriate to install sensor units in combination with, for example, kinetic converters shown in Fig. 5.

\section{Energy Supply}

Various conversion principles with different power ratings are used to meet the energy requirements of different application scenarios. These include, for example, electrodynamic converters which can, for example, be fitted via adapters to the front end of a handling unit in order to exploit acceleration energy and provide a local electrical energy supply. A solution developed within the research project "MIKOA" is shown in Fig. 5. All converter modules show the same mechanical dimensions. This is part of a modular concept for the complete variety of imple4mented converters. 


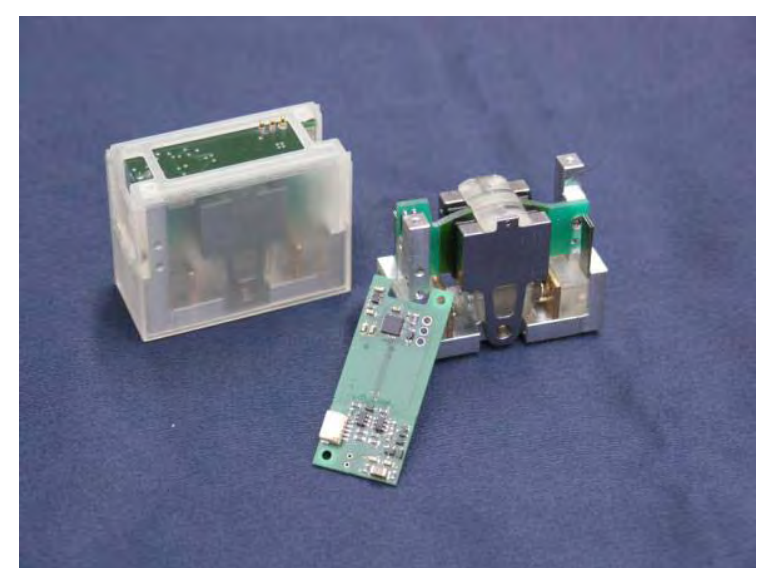

Fig. 5 Dynamic converter (source: HSG IMIT)

Kinetic converters of this kind are generally activated by impact or shock energy, (occasionally, pulsed or in vibration) and can be of harmonic or discontinuous design. The power levels which can be achieved vary between a few $\mu \mathrm{W}$ and several $100 \mathrm{~mW}$ and depend heavily on the conversion principle used and the moving vibration mass. Inductive, capacitive or piezoelectric conversion methods can be used. In selecting a method, special attention has to be paid on the achievable output voltages and their suitability for use. In general, kinetic generators need to be matched to specific application cases. An ideal generator can be selected for a given application with the aid of simulation tools on the basis of known dynamic activation profiles.

Amorphous solar cells and so-called P/E converters are also used in order to provide a continuous electrical power supply in low-power operation. They offer a large bandwidth of usable light energy, which is important particularly for indoor applications with artificial light. A further major goal of the project is the development of a solar cell energy management system which allows, among other things, maximum power point tracking. This involves a continuous adjustment of the working point as a function of radiation intensity and temperature.

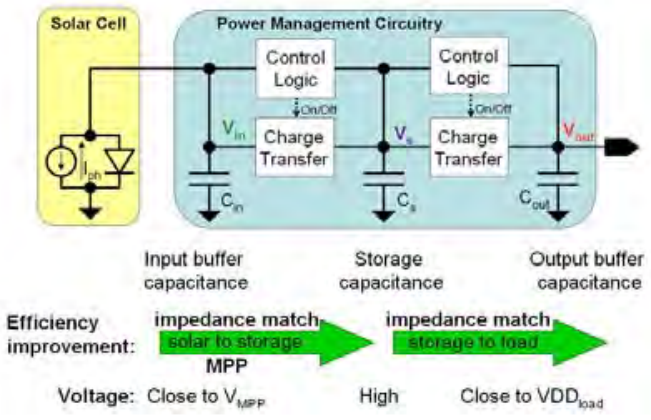

Fig. 6 Block Diagramm of Solar Powermanagement IC (source: Siemens)
The most important conversion principle in terms of power output makes use of the fluidic pressure and flow energy from the compressed air supply system or the residual energy from the exhaust air of pneumatic systems. In pneumatic lamellar motors or turbines, this fluidic energy is converted into rotary motion energy, which is then converted by generators into electrical energy using the inductive principle.

The general physical difference between pneumatic lamellar motors and turbines is that with the former, drive torque is generated by the presence of a differential pressure, while in the case of turbines it is generated by the aerodynamic impact of the flowing medium on an impeller wheel. Due to the differences in physical operating principles, the power output and efficieny of turbines is only comparable to pneumatic lamellar motors when considerably higher rotary speeds are present, leading to special requirements concerning the generator design. On the other hand, the fact that for turbines, the differential pressure required for starting is relatively low, which means that they can easily be operated with the exhaust air of pneumatic systems, allowing exploitation of unused residual energy without significant reactive effects on the system.

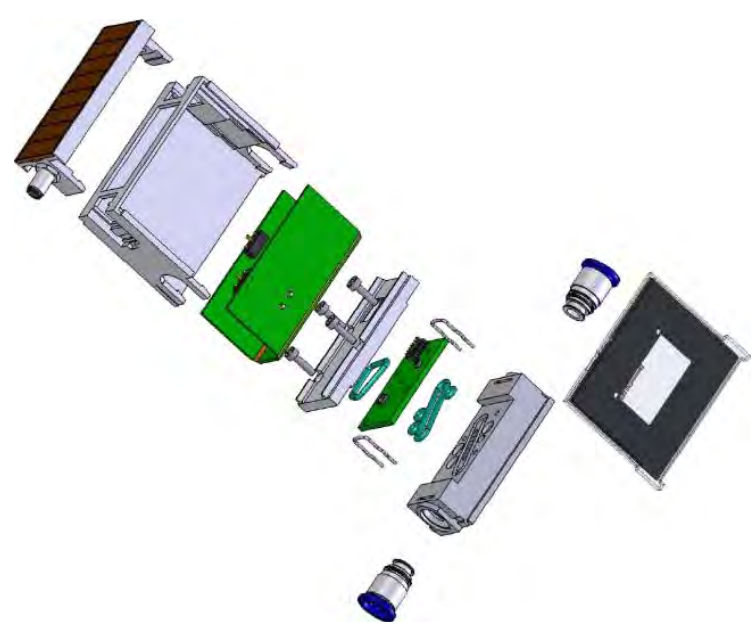

Fig. 7 Design concept for a modular sensor node (source: HSG-IMIT; Zollner)

The achievable power output of the lamellar motor generator unit developed in the project reaches the double-digit wattage range while largely operating in an intermittent scenario. This means that the unit, when served from the compressed air supply network for a certain time, delivers considerably more power than the application requires. This surplus of power is buffered in an energy reservoir which can be accessed by the application whenever the conversion process is not operating. Once a defined quantity of energy has been drawn from 
the energy reservoir, the conversion process is re-started.

The turbine generator unit behaves differently. Depending on its geometrical design, power is delivered in a low double-digit wattage range. As this type of converter unit is generally installed in the exhaust air of pneumatic systems, the level of flow energy applied may vary widely. This characteristic should be taken into account when the dimensioning of the system is made.

\section{Reliable Wireless Communication}

The parameters of the addressed automation areas make wireless communication one of the greatest challenges of the project. Two different wireless protocols are used to accommodate varying needs. For fast deterministic and reliable communication as required in handling processes, a proprietary protocol in the $2.4 \mathrm{GHz}$ band is used. This protocol has been especially developed and optimized for this kind of applications and is useful for local field communication in production cells. It provides narrow-band frequency-agile data transmission. In simple terms, it is able to avoid conflicts with other wireless systems by using the frequencyhopping method and thus co-exists with these without collisions. At the same time, it imposes only a very slight burden on the medium "wireless channel". The typical latency period for a sampling of over 100 wireless devices is less than $10 \mathrm{~ms}$, making it possible to conform to the real-time criteria customary in the automation sector. Thanks to the implemented safety mechanisms, the typical packet loss rate in data transmission is $1 \mathrm{e}-9$, an order of magnitude which is comparable to a wired connection. This characteristic variable is the ratio of lost data packets to transmitted data packets. In order to illustrate the ability of this protocol to co-exist with WLAN, Fig. 8 shows its narrow-band transmission in the bandwidth gaps between the WLAN frequency bands.

For non-time-critical monitoring functions in complete production installations, a protocol based on a frequency of $868 \mathrm{MHz}$ is used, allowing communication over larger distances up to $100 \mathrm{~m}$.

As time requirements in this application field are relatively undemanding and a low level of reliability has no safety-critical consequences, it is permissible to use short telegrams. Assuming a low probability of collisions, energy-intensive safety measures are absent. This allows wireless communication with very low energy consumption.

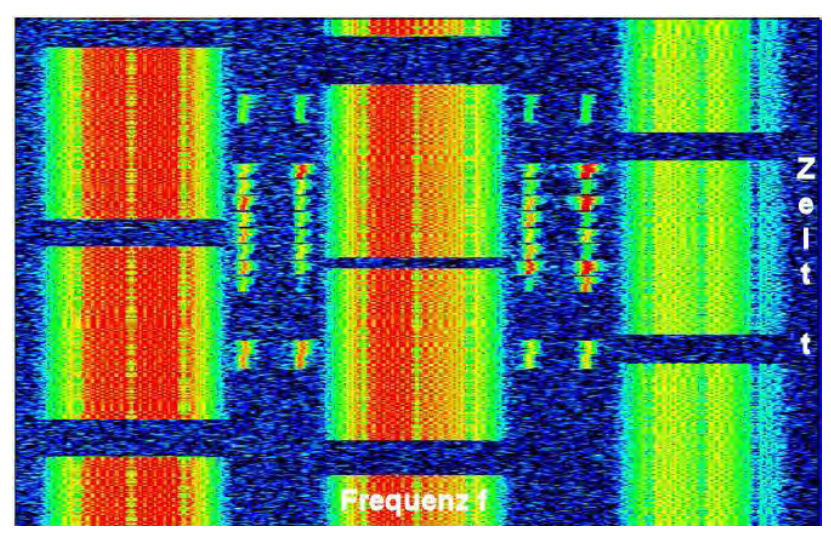

Fig. 8 Frequency/time graph (source: HSU)

Both scenarios can be met by a wireless base station that was developed to receive both protocols and process the relevant data. This is shown in Fig. 9.

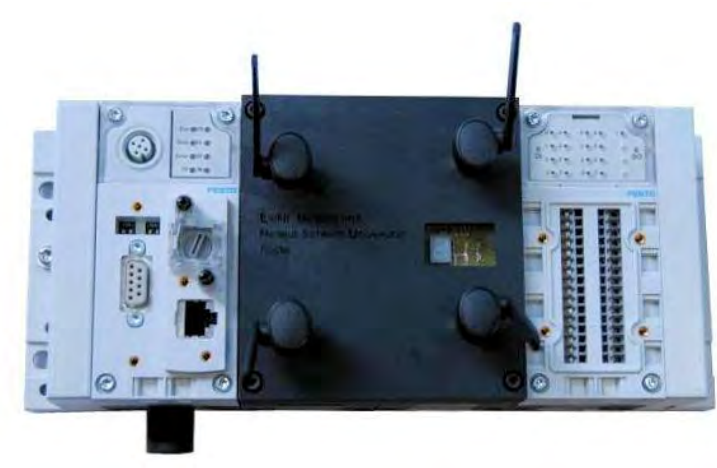

Fig. 9 Wireless base station (source: HSU/Festo)

\section{Summary}

Considering the increased complexity and the large volumes of data involved, the factory installations of the future will require new groundbreaking concepts which allow a better management of manufacturing and handling processes. The composite project MIKOA, with its goal to develop miniaturized autonomously-operating sensor and actuator systems which communicate wireless, is able to make major contributions to these concepts. The emphasis of MIKOA is on miniaturization of all necessary function modules and the development of suitable wireless modules for the implementation of reliable and energyoptimized transmission methods.

\section{References}

[1] MIKOA "Miniaturised self-sufficient components with reliable wireless communication for automation systems". This is a public funded research project which forms part of the microsystems research program of the Federal German Ministry of Education and Research (BMBF); www.mikoa.de

[2] „EcoStruxure“ Schneider Electric 